\title{
双官能膦配体-金(I)高效催化合成 1,5-苯并二氮草
}

\author{
郑康河周丙伟金红卫 刘运奎* \\ (浙江工业大学化学工程学院 绿色化学与技术国家重点实验室培育基地 杭州 310014)
}

\begin{abstract}
摘要 在室温条件下，使用双官能膦-金(I)催化剂催化邻苯二胺与炔烃进行亲核加成/环化反应，一步合成 1,5-苯并二 氮草类化合物. 该反应具有原子经济性高、原料简单易得、操作便捷及反应温和等优点.

关键词 邻苯二胺; 炔烃; 双官能膦配体; 金催化; 1,5-苯并二氮草
\end{abstract}

\section{Bifunctional Phosphine Ligand-Enabled Gold(I)-Catalyzed Efficient Synthesis of 1,5-Benzodiazepines}

\author{
Zheng, Kanghe Zhou, Bingwei Jin, Hongwei Liu, Yunkui* \\ (State Key Laboratory Breeding Base of Green Chemistry-Synthesis Technology, College of Chemical Engineering, \\ Zhejiang University of Technology, Hangzhou 310014)
}

\begin{abstract}
At room temperature, a bifunctional phosphine-gold(I) catalyst was used to catalyze the nucleophilic addition/ cyclization reaction of $o$-phenylenediamines with alkynes to generate 1,5-benzodiazepines in one step. The reaction has the advantages of high atomic-economy, simple raw materials, convenient operation and mild reaction conditions.

Keywords phthaline diamine; alkyne; bifunctional phosphine ligand; gold catalysis; 1,5-benzodiazepine
\end{abstract}

苯并二氮草类化合物是一类重要的具有药理活性 的杂环化合物，具有镇静、催眠、抗炎、抗神经性等功 能，在医药领域有着广泛的应用 ${ }^{[1]}$. 例如，用于治疗精 神分裂症的氯氮平和洛沙平 ${ }^{[2]}$, 以及 HIV (human immunodeficiency virus)衣壳装配抑制剂[3]等都具有苯并二 氮草骨架. 1,5-苯并二氮草类化合物不仅本身具有潜在 的药理活性, 而且还可作为一些稠环类化合物的重要合 成中间体 ${ }^{[4]}$. 因此, 研究简便、高效的苯并二氮草类化合 物的合成方法具有重要的意义.

1,5-苯并二氮草类化合物传统的合成方法主要是通 过邻苯二胺与 $\alpha, \beta$-不饱和酮 ${ }^{[5]} 、 \beta$-卤代酩同 ${ }^{[6]}$ 或者酮类 ${ }^{[7]}$ 化合物进行缩合反应. 但是, 此类缩合方法原子经济性 偏低. 鉴于炔烃是制备酮类化合物的前体, 因此, 如果 能够直接以炔烃作为底物来合成苯并二氮草类化合物, 不仅能够解决步骤经济性问题, 而且能够有效提高反应 的原子经济性. 2012 年, Maiti 等 ${ }^{[8]}$ 报道了邻苯二胺与炔 烃在 $\mathrm{HgOTf}_{2}$ 催化下合成 1,5 -苯并二氮草. 这个方法实
现了更为原子经济性的合成, 但却使用了高毒的含录催 化剂. 2012 年我们课题组 ${ }^{[9]}$ 以及 2014 年 Luo 课题组 ${ }^{[10]}$ 分别报道了使用金催化剂来替代录催化剂, 实现了由邻 苯二胺与炔烃直接合成苯并二氮草类化合物, 但都需要 较高的反应温度. 我们发现在较高的温度下, 金催化剂 容易失活，从而影响金的催化效率. 这种在较高温度下 金催化剂容易失活的现象是金催化反应中较为常见的 问题, 尤其是在有胺类化合物参与的加成反应中更易发 生 ${ }^{[11-12]}$. 因此, 如何通过配体的设计提高金催化剂的活 性和稳定性，避免金催化剂失活一直是金催化领域受到 关注的课题.

近些年来, “受阻路易斯酸碱对” 理论受到了化学 界的广泛关注 ${ }^{[13]}$. 具有 “受阻路易斯酸碱对” 的分子中 同时存在路易斯酸和路易斯碱活化中心，它们能够对小 分子的化学键进行协同 “推拉式” (push and pull)活化作 用, 从而能够加速化学键的断裂. 受 “受阻路易斯酸碱 对” 理论启发, Zhang 课题组 ${ }^{[14]}$ 巧妙地把这种理念运用

\footnotetext{
* Corresponding author. E-mail: ykuiliu@zjut.edu.cn

Received March 9, 2020; revised May 28, 2020; published online June 8, 2020.

Project supported by the National Natural Science Foundation of China (Nos. 21772176, 21372201), and the Natural Science Foundation of Zhejiang Province (No. LY20B020013)

国家自然科学基金(Nos. 21772176, 21372201)和浙江省自然科学基金(No. LY20B020013)资助项目.
} 
到具有 Lewis 酸/碱双活化中心的金催化剂的设计上. 在 这些催化剂中, 他们使用的是一类结构新颖的联苯型膦 配体. 与常用的 Buchwald 膦配体不同, 这些新型配体仍 以 2-联苯基膦为骨架, 但都在其悬挂的苯环下半部分安 装有独特的碱性官能团. 当与金配位之后, 所形成的金 催化剂既具有以金为中心的 Lewis 酸活化中心, 同时又 具有以碱性基团为中心的 Lewis 碱活化中心. 因此, 在 具体反应中, 利用一价金络合物 [配体-金(I)-底物]的独 特的线性结构(Scheme 1a) 能形成受阻 Lewis 酸碱对, 从 而实现这些碱性官能团和亲核试剂之间的有益相互作 用，达到催化体系对反应过渡态的双重协同活化作用， 从而可以极大地提高金的催化活性、降低金的催化用量 以及实现温和条件下的金催化反应. 基于以上背景及在 金催化方面的兴趣 ${ }^{[9,15]}$, 我们在此报道一例双官能化膦 配体金(I)催化邻苯二胺与炔烃的亲核加成/环化反应, 于室温条件下高效合成了 1,5 -苯并二氮草类化合物 (Scheme 1b).

\section{1 结果与讨论}

\section{1 反应条件的优化}

首先, 以邻苯二胺和苯乙炔为模板底物, 优化了合 适的反应条件(表 1). 以 $\mathrm{Ph}_{3} \mathrm{PAuNTf}_{2}$ (5 mol\%)为催化剂, 在 $\mathrm{CHCl}_{3}$ 中于室温条件下反应 $24 \mathrm{~h}$, 只得到 $42 \%$ 的收率 (Entry 1, 表 1). 即便延长反应时间也不能再提高转化 率, 表明金催化剂可能已经失活. 当使用氮杂环卡宾配 体配位的金催化剂, 如 IMesAuNTf 2 和 IPrAuNTf 2 时, 其 产率大致相同(Entries 2, 3, 表 1). 当使用 Zhang 课题组 合成的具有远端酰胺碱性基团的 WangPhos (L1)AuNTf $_{2}{ }^{[14 a]}$ 时(Scheme 1a), 反应 $12 \mathrm{~h}$ 收率显著提高到 87\% (Entry 4, 表 1). 通过系统篮选配位阴离子、溶剂、
催化剂用量及反应时间(Entries 4 12, 表 1), 最终发现 以 $\mathrm{L1AuNTf}_{2}$ (5 mol\%)为催化剂在二氯甲烷中反应 $6 \mathrm{~h}$, 可以获得 $93 \%$ 产率的目标化合物(Entry 9, 表 1). 作为对 比，当使用同样为联苯型膦配体但没有远端酰胺基团的 Ad-JohnPhos 时, 目标化合物产率要低很多(Entry 13 vs. 9, 表 1). 其他一价和三价金催化剂及 $\mathrm{ZnCl}_{2}, \mathrm{FeCl}_{3}$ 等 Lewis 酸催化剂, 对反应没有催化效果(Entries $14 \sim 17$, 表 1). 控制实验表明，没有金催化剂，该反应不能进行 (Entry 18，表 1).

\section{2 底物的拓展}

在获得最优反应条件后，首先对邻苯二胺 $\mathbf{1}$ 的普适 性进行了考察. 如表 2 所示, 从一系列不同取代基的邻 苯二胺类化合物 $(\mathbf{1} \mathbf{a} \sim 1 \mathrm{e}$, 表 2)来看, 取代基为供电子基 团反应效果优于吸电子基团(Entries 2, 3 vs. 4, 5, 表 2). 接下来对炔烃 2 的普适性进行考察. 芳香基末端炔烃, 不管是给电子基团还是吸电子基团取代的炔烃都可以 顺利与邻苯二胺反应, 得到较高产率的产物(Entries 6 16, 表 2), 其中给电子基团取代的比吸电子基团取代的 芳炔反应效果要好. 此外, 杂环类如噻吩取代的末端炔 烃与邻苯二胺反应也很顺利, 得到 $56 \%$ 产率的目标产物 (Entry 17, 表 2). 最后对烷基末端炔烃和内炔也进行了 考察, 发现烷基末端炔烃也能参与反应, 但效果相对较 差(Entries 18，19，表 2)，但内炔不能参与反应(Entry 20, 表 2). 当使用 $1 \mathrm{mmol}$ 规模 $\mathbf{1 a}$ 时, 模板反应也能取得 90\%产率 (Entry 1，表 2).

根据相关文献报道 ${ }^{[9-10,14]}$, 我们提出了该反应的可 能机理(Scheme 2). 在胺与炔烃的亲核加成过程中, 由 于金催化剂中远端的酰胺基团可以通过氢键与胺基发 生作用，使得该亲核加成反应有类似 “准分子内亲核加
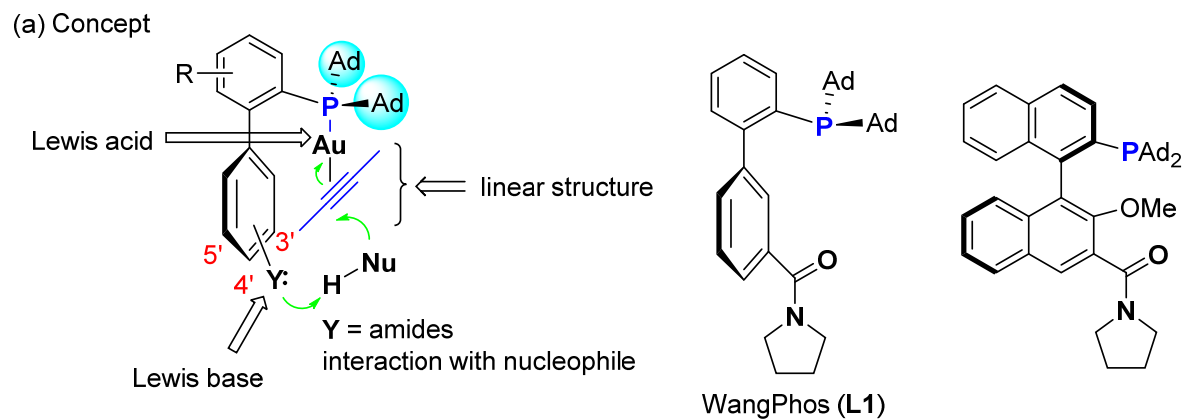

(b) Application in the synthesis of 1,5-benzodiazepines from o-phenylenediamines with alkynes at room temperature<smiles>[R]c1cc(N)c(N)cc1[R]</smiles>
$2 \mathrm{R}^{2}=$

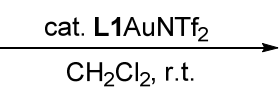<smiles>[R]C1=Nc2cc([R])c([R])cc2NC([R])(C)C1</smiles>

图式 1 双官能膦-金(I)高效催化亲核试剂对炔烃加成示意图及其应用于合成 1,5-二氮杂草

Scheme 1 Illustration of gold(I)-catalyzed highly efficient activation of the addition of nucleophiles across alkynes enabled by bifunctional phosphine ligands and application in the synthesis of 1,5-benzodiazepines 
表 1 反应条件的优化 ${ }^{a}$

Table 1 Optimization of the reaction conditions

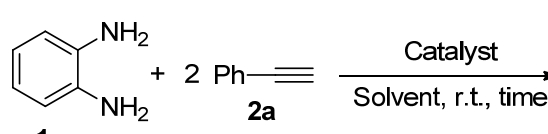<smiles>CC1(c2ccccc2)CC(c2ccccc2)=Nc2ccccc2N1</smiles>

3aa

\begin{tabular}{|c|c|c|c|c|}
\hline Entry & Catalyst (mol\%) & Solvent & Time/h & Yield $^{b} / \%$ \\
\hline 1 & $\mathrm{Ph}_{3} \mathrm{PAuNTf}_{2}(5.0)$ & $\mathrm{CHCl}_{3}$ & 24 & 42 \\
\hline 2 & $\operatorname{IMesAuNTf}_{2}(5.0)$ & $\mathrm{CHCl}_{3}$ & 24 & 45 \\
\hline 3 & $\operatorname{IPrAuNTf}_{2}(5.0)$ & $\mathrm{CHCl}_{3}$ & 24 & 46 \\
\hline 4 & $\mathbf{L 1 A u N T f}_{2}(5.0)$ & $\mathrm{CHCl}_{3}$ & 12 & 87 \\
\hline 5 & $\mathbf{L 1 A u O T f}_{2}(5.0)$ & $\mathrm{CHCl}_{3}$ & 12 & 84 \\
\hline 6 & $\mathbf{L 1 A u S b F}_{6}(5.0)$ & $\mathrm{CHCl}_{3}$ & 12 & 85 \\
\hline 7 & $\mathbf{L 1 A u N T f}_{2}(5.0)$ & DCE & 12 & 90 \\
\hline 8 & $\mathbf{L 1 A u N T f}_{2}(5.0)$ & Toluene & 12 & 84 \\
\hline 9 & $\operatorname{L1AuNTf}_{2}(5.0)$ & $\mathrm{CH}_{2} \mathrm{Cl}_{2}$ & 6 & 93 \\
\hline 10 & $\mathbf{L 1 A u N T f}_{2}(5.0)$ & $\mathrm{CH}_{2} \mathrm{Cl}_{2}$ & $4 / 8$ & $85 / 93$ \\
\hline 11 & $\mathbf{L 1 A u N T f}_{2}(1.0)$ & $\mathrm{CH}_{2} \mathrm{Cl}_{2}$ & 12 & 69 \\
\hline 12 & $\mathbf{L 1 A u N T f}_{2}(3.0)$ & $\mathrm{CH}_{2} \mathrm{Cl}_{2}$ & 12 & 87 \\
\hline 13 & Ad-JohnPhosAuNTf 2 (5.0) & $\mathrm{CH}_{2} \mathrm{Cl}_{2}$ & 6 & 48 \\
\hline 14 & $\mathrm{AuCl} / \mathrm{AgNTf}_{2}(5.0)$ & $\mathrm{CH}_{2} \mathrm{Cl}_{2}$ & 12 & Trace \\
\hline 15 & $\mathrm{AuCl}_{3} / 3 \mathrm{AgNTf}_{2}(5.0)$ & $\mathrm{CH}_{2} \mathrm{Cl}_{2}$ & 12 & - \\
\hline 16 & $\mathrm{ZnCl}_{2}(5.0)$ & $\mathrm{CH}_{2} \mathrm{Cl}_{2}$ & 12 & - \\
\hline 17 & $\mathrm{FeCl}_{3}(5.0)$ & $\mathrm{CH}_{2} \mathrm{Cl}_{2}$ & 12 & - \\
\hline 18 & None & $\mathrm{CH}_{2} \mathrm{Cl}_{2}$ & 12 & - \\
\hline
\end{tabular}

${ }^{a}$ Reaction conditions: $1 \mathrm{a}(0.2 \mathrm{mmol})$ and $\mathbf{2 a}(0.5 \mathrm{mmol})$, catalyst, solvent $(0.5$ $\mathrm{mL}), 25{ }^{\circ} \mathrm{C}$ under air unless otherwise noted. ${ }^{b}$ Yield was determined by HPLC analysis using diphenyl as an internal standard.
表 2 邻苯二胺类化合物 $\mathbf{1}$ 和炔烃 $\mathbf{2}$ 的拓展 ${ }^{a}$

Table 2 Substrate scope for phthaline diamine 1 and alkyne 2

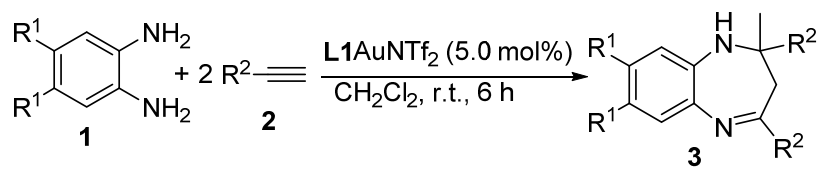

\begin{tabular}{|c|c|c|c|c|}
\hline Entry & $\mathrm{R}^{1}$ & $\mathrm{R}^{2}$ & Product & Yield $^{b} / \%$ \\
\hline 1 & $\mathrm{H}(\mathbf{1 a})$ & $\mathrm{Ph}$ & $3 \mathbf{a a}$ & $92\left(90^{c}\right)$ \\
\hline 2 & $\mathrm{Me}(\mathbf{1 b})$ & $\mathrm{Ph}$ & $3 \mathbf{b a}$ & 92 \\
\hline 3 & $\mathrm{MeO}(\mathbf{1 c})$ & $\mathrm{Ph}$ & $3 \mathrm{ca}$ & 94 \\
\hline 4 & $\mathrm{Cl}(\mathbf{1 d})$ & $\mathrm{Ph}$ & 3da & 70 \\
\hline 5 & $\operatorname{Br}(1 \mathrm{e})$ & $\mathrm{Ph}$ & 3ea & 63 \\
\hline 6 & $\mathrm{H}(\mathbf{1 a})$ & $4-\mathrm{FC}_{6} \mathrm{H}_{4}(\mathbf{2 b})$ & $3 \mathbf{a b}$ & 93 \\
\hline 7 & $\mathrm{H}$ & $4-\mathrm{ClC}_{6} \mathrm{H}_{4}(\mathbf{2 c})$ & $3 a c$ & 94 \\
\hline 8 & $\mathrm{H}$ & $4-\mathrm{BrC}_{6} \mathrm{H}_{4}(\mathbf{2 d})$ & 3ad & 96 \\
\hline 9 & $\mathrm{H}$ & $4-\mathrm{NCC}_{6} \mathrm{H}_{4}(\mathbf{2 e})$ & 3ae & 56 \\
\hline 10 & $\mathrm{H}$ & $4-\mathrm{O}_{2} \mathrm{NC}_{6} \mathrm{H}_{4}(\mathbf{2 f})$ & 3 af & 45 \\
\hline 11 & $\mathrm{H}$ & $4-\mathrm{MeOCOC}_{6} \mathrm{H}_{4}(\mathbf{2 g})$ & $3 a g$ & 82 \\
\hline 12 & $\mathrm{H}$ & $4-\mathrm{MeOC}_{6} \mathrm{H}_{4}(\mathbf{2 h})$ & 3ah & 80 \\
\hline 13 & $\mathrm{H}$ & $4-\mathrm{MeC}_{6} \mathrm{H}_{4}(\mathbf{2 i})$ & 3ai & 90 \\
\hline 14 & $\mathrm{H}$ & $4-\mathrm{PhC}_{6} \mathrm{H}_{4}(\mathbf{2} \mathbf{j})$ & 3aj & 84 \\
\hline 15 & $\mathrm{H}$ & 4- $\mathrm{PhCOC}_{6} \mathrm{H}_{4}(\mathbf{2 k})$ & 3ak & 86 \\
\hline 16 & $\mathrm{H}$ & $3-\mathrm{BrC}_{6} \mathrm{H}_{4}(\mathbf{2 l})$ & 3al & 74 \\
\hline 17 & $\mathrm{H}$ & 2-Thienyl (2m) & 3am & 56 \\
\hline 18 & $\mathrm{H}$ & Cyclopropyl (2n) & 3an & 40 \\
\hline 19 & $\mathrm{H}$ & $n-\mathrm{C}_{6} \mathrm{H}_{13}(\mathbf{2 o})$ & $3 \mathbf{a o}$ & 47 \\
\hline 20 & $\mathrm{H}$ & But-1-yn-1-ylbenzene (2p) & 3ap & 0 \\
\hline
\end{tabular}

${ }^{a}$ Reaction conditions: $1(0.2 \mathrm{mmol})$ and $\mathbf{2}(0.5 \mathrm{mmol}), \mathbf{L 1} \mathrm{AuNTf}_{2}(5.0 \mathrm{~mol} \%)$ $\mathrm{CH}_{2} \mathrm{Cl}_{2}(0.5 \mathrm{~mL}), 25{ }^{\circ} \mathrm{C}$ for $6 \mathrm{~h}$ under air unless otherwise noted. ${ }^{b}$ Isolated yields. ${ }^{c}$ The yield was obtained when using $1 \mathrm{mmol}-$ scale of $\mathbf{1 a}$.

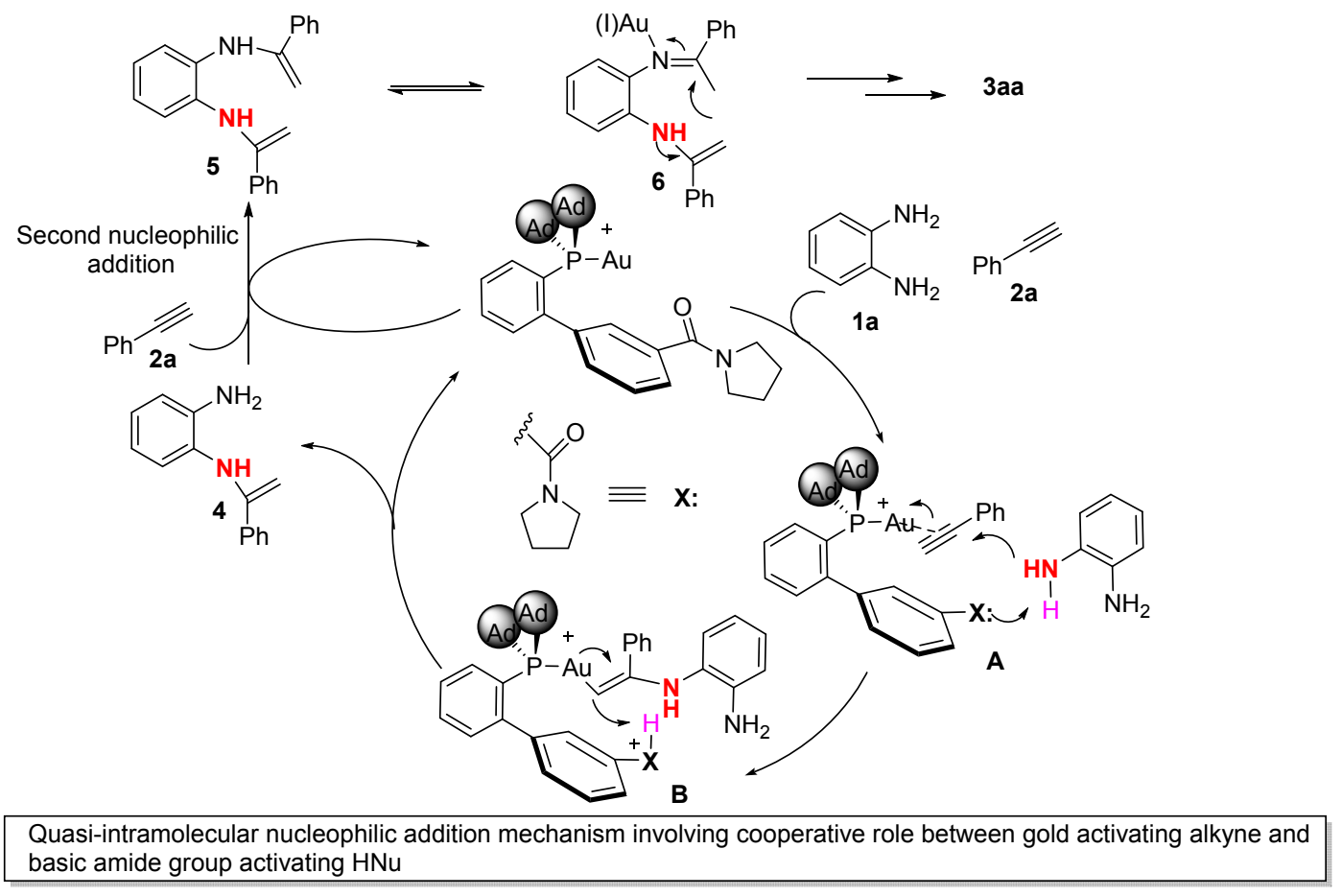

图式 2 可能的反应机理

Scheme 2 Proposed mechanism 
成反应” (quasi-intramolecular nucleophilic addition)的 性质 ${ }^{[14]}$, 从而有利于反应的加速进行.

\section{2 结论}

通过使用具有远端碱性酰胺基团的联苯型膦配体 (WangPhos, L1), 实现了在室温条件下金(I)催化的邻苯 二胺类化合物和炔烃的亲核加成/环化反应，高效构建 了 1,5 - 苯并二氮草类化合物. 该反应具有原子经济性 高、原料易得、操作简单、反应条件温和等优点, 且可 有效避免金催化剂的失活现象.

\section{3 实验部分}

\section{1 仪器与试剂}

${ }^{1} \mathrm{H}$ NMR 和 ${ }^{13} \mathrm{C}$ NMR 均在德国 Bruker 公司 $500 \mathrm{MHz}$ 核磁共振仪上测定, $\mathrm{CDCl}_{3}$ 为溶剂, TMS 为内标; 柱色谱 分离使用 $100 \sim 200$ 目硅胶, 以石油醚和乙酸乙酯为洗 脱剂. 实验所用试剂均购于 Aladdin 试剂公司.

\section{2 实验方法}

在 $10 \mathrm{~mL}$ 反应管中加入邻苯二胺 $(0.2 \mathrm{mmol})$ 、苯乙 炔 $(0.5 \mathrm{mmol}) 、$ 金催化剂 $(5.0 \mathrm{~mol} \%)$ 和二氯甲烷 $(0.5 \mathrm{~mL})$, 然后在室温搅拌下反应 $6 \mathrm{~h}$. 停止反应后, 反应液中加 入 $100 \sim 200$ 目硅胶, 减压除去溶剂, 然后用柱层析分离 提纯, 洗脱剂为石油醚和乙酸乙酯 $(V / V=10 / 1)$, 得到 1,5-苯并二氮草类化合物.

2,3-二氢-2-甲基-2,4-二苯基- $1 H-1,5$-二苯并二氮草 (3aa): 产率 $92 \%$. 黄色固体, m.p. $150 \sim 152{ }^{\circ} \mathrm{C}$ (lit. ${ }^{[9]}$ m.p. $\left.150 \sim 152{ }^{\circ} \mathrm{C}\right) ;{ }^{1} \mathrm{H}$ NMR $\left(500 \mathrm{MHz}, \mathrm{CDCl}_{3}\right) \delta$ : 7.66 (dd, $J=7.3,1.2 \mathrm{~Hz}, 4 \mathrm{H}), 7.44 \sim 7.21(\mathrm{~m}, 7 \mathrm{H}), 7.15 \sim 7.09$ (m, 2H), 6.88 (dd, $J=7.4,1.7 \mathrm{~Hz}, 1 \mathrm{H}), 3.57$ (s, 1H), 3.19 (d, $J=13.2 \mathrm{~Hz}, 1 \mathrm{H}), 3.02$ (d, $J=13.2 \mathrm{~Hz}, 1 \mathrm{H}), 1.80$ (s, $3 \mathrm{H}) ;{ }^{13} \mathrm{C}$ NMR $\left(126 \mathrm{MHz}, \mathrm{CDCl}_{3}\right) \delta: 167.5,147.5,140.0$, $139.5,138.0,129.6,128.6,128.2,127.9,127.0,127.0$, 126.3, 125.4, 121.5, 121.4, 73.5, 43.0, 29.8.

2,3-二氢-2,7,8-三甲基- 2,4 -二苯基- $1 H-1,5$-苯并二氮 草(3ba): 产率 92\%. 黄色固体, m.p. $135 \sim 138{ }^{\circ} \mathrm{C}$ (lit. ${ }^{[9]}$ m.p. $\left.137 \sim 139{ }^{\circ} \mathrm{C}\right) ;{ }^{1} \mathrm{H}$ NMR $\left(500 \mathrm{MHz}, \mathrm{CDCl}_{3}\right) \delta$ : $7.66 \sim 7.54(\mathrm{~m}, 4 \mathrm{H}), 7.32 \sim 7.27(\mathrm{~m}, 2 \mathrm{H}), 7.27 \sim 7.22(\mathrm{~m}$, $3 \mathrm{H}), 7.21 \sim 7.17(\mathrm{~m}, 1 \mathrm{H}), 7.14(\mathrm{~s}, 1 \mathrm{H}), 6.66(\mathrm{~s}, 1 \mathrm{H}), 3.44$ (s, 1H), $3.14(\mathrm{~d}, J=13.2 \mathrm{~Hz}, 1 \mathrm{H}), 2.98(\mathrm{~d}, J=13.2 \mathrm{~Hz}$, 1H), 2.27 (d, $J=1.6 \mathrm{~Hz}, 6 \mathrm{H}), 1.76$ (s, 3H); ${ }^{13} \mathrm{C}$ NMR $(126$ $\left.\mathrm{MHz}, \mathrm{CDCl}_{3}\right) \delta: 166.9,147.9,139.9,137.7,135.9,134.9$, $129.7,129.6,129.5,128.3,128.0,127.0,127.0,125.5$, 122.3, 73.2, 43.3, 29.91, 19.4, 18.8.

7,8-二甲氧基-2,3-二氢-2-甲基-2,4-二苯基- $1 H-1,5$ 苯并二氮草(3ca): 产率 94\%. 橙红色油状物; ${ }^{1} \mathrm{H}$ NMR $\left(500 \mathrm{MHz}, \mathrm{CDCl}_{3}\right) \delta: 7.63 \sim 7.57(\mathrm{~m}, 4 \mathrm{H}), 7.33 \sim 7.17(\mathrm{~m}$, $6 \mathrm{H}), 6.94(\mathrm{~s}, 1 \mathrm{H}), 6.39(\mathrm{~s}, 1 \mathrm{H}), 3.89(\mathrm{~s}, 6 \mathrm{H}), 3.40(\mathrm{~s}, 1 \mathrm{H})$, 3.17 (d, $J=13.2 \mathrm{~Hz}, 1 \mathrm{H}), 2.98(\mathrm{~d}, J=13.2 \mathrm{~Hz}, 1 \mathrm{H}), 1.75$ (s, 3H); ${ }^{13} \mathrm{C}$ NMR $\left(126 \mathrm{MHz}, \mathrm{CDCl}_{3}\right) \delta: 166.7,147.6$, $147.4,144.1,139.8,133.1,131.6,129.4,128.2,128.0$, 127.0, 126.8, 125.3, 112.0, 104.9, 73.8, 56.1, 56.1, 43.4, 29.8; HRMS (ESI) calcd for $\mathrm{C}_{24} \mathrm{H}_{25} \mathrm{~N}_{2} \mathrm{O}_{2}[\mathrm{M}+\mathrm{H}]^{+}$ 373.1911, found 373.1919.

7,8-二氯-2,3-二氢-2-甲基-2,4-二苯基- $1 H$ - 1,5 -苯并 二氮草(3da): 产率 $70 \%$. 黄色固体, m.p. $159 \sim 161{ }^{\circ} \mathrm{C}$ (lit. ${ }^{[10]}$ m.p. $\left.161 \sim 162{ }^{\circ} \mathrm{C}\right) ;{ }^{1} \mathrm{H}$ NMR $\left(500 \mathrm{MHz}, \mathrm{CDCl}_{3}\right) \delta$ : $7.58 \sim 7.55(\mathrm{~m}, 4 \mathrm{H}), 7.43(\mathrm{~s}, 1 \mathrm{H}), 7.37 \sim 7.31(\mathrm{~m}, 1 \mathrm{H})$, $7.31 \sim 7.18(\mathrm{~m}, 5 \mathrm{H}), 6.93(\mathrm{~s}, 1 \mathrm{H}), 3.62(\mathrm{~s}, 1 \mathrm{H}), 3.19(\mathrm{~d}, J=$ $13.4 \mathrm{~Hz}, 1 \mathrm{H}), 2.98(\mathrm{~d}, J=13.5 \mathrm{~Hz}, 1 \mathrm{H}), 1.77(\mathrm{~s}, 3 \mathrm{H}) ;{ }^{13} \mathrm{C}$ NMR $\left(126 \mathrm{MHz}, \mathrm{CDCl}_{3}\right) \delta: 168.7,146.8,139.2,139.0$, $137.8,130.2,129.9,128.8,128.4,128.1,127.3,127.1$, $125.3,124.1,121.8,72.8,43.2,29.9$.

7,8-二溴-2,3-二氢-2-甲基-2,4-二苯基- $1 H$ - 1,5 -苯并 二氮草(3ea): 产率 63\%. 橙色油状物; ${ }^{1} \mathrm{H}$ NMR (500 $\left.\mathrm{MHz}, \mathrm{CDCl}_{3}\right) \delta: 7.59 \sim 7.52(\mathrm{~m}, 5 \mathrm{H}), 7.36 \sim 7.31(\mathrm{~m}, 1 \mathrm{H})$, $7.30 \sim 7.18(\mathrm{~m}, 5 \mathrm{H}), 7.12(\mathrm{~s}, 1 \mathrm{H}), 3.64(\mathrm{~s}, 1 \mathrm{H}), 3.20$ (d, $J=$ $13.4 \mathrm{~Hz}, 1 \mathrm{H}), 2.98(\mathrm{~d}, J=13.5 \mathrm{~Hz}, 1 \mathrm{H}), 1.78(\mathrm{~s}, 3 \mathrm{H}) ;{ }^{13} \mathrm{C}$ NMR $\left(126 \mathrm{MHz}, \mathrm{CDCl}_{3}\right) \delta: 168.7,146.8,139.8,139.1$, $138.5,133.1,130.2,128.5,128.1,127.4,127.2,125.3$, 124.9, 120.9, 115.3, 72.8, 43.3, 30.1; HRMS (ESI) calcd for $\mathrm{C}_{22} \mathrm{H}_{19} \mathrm{Br}_{2} \mathrm{~N}_{2}[\mathrm{M}+\mathrm{H}]^{+}$468.9910, found 468.9915.

2,4-双(4-氟苯基)-2,3-二氢-2-甲基- $1 H$ - 1,5 -苯并二氮 草(3ab): 产率 93\%. 黄色固体, m.p. 104 106 ${ }^{\circ} \mathrm{C}$ (lit. ${ }^{[0]}$ m.p. $\left.106 \sim 107{ }^{\circ} \mathrm{C}\right) ;{ }^{1} \mathrm{H}$ NMR $\left(500 \mathrm{MHz}, \mathrm{CDCl}_{3}\right) \delta$ : $7.63 \sim 7.52(\mathrm{~m}, 4 \mathrm{H}), 7.36 \sim 7.30(\mathrm{~m}, 1 \mathrm{H}), 7.13 \sim 7.05(\mathrm{~m}$, $2 \mathrm{H}), 6.96 \sim 6.90(\mathrm{~m}, 4 \mathrm{H}), 6.88 \sim 6.82(\mathrm{~m}, 1 \mathrm{H}), 3.43(\mathrm{~s}$, 1H), 3.10 (d, $J=13.2 \mathrm{~Hz}, 1 \mathrm{H}), 2.91$ (d, $J=13.3 \mathrm{~Hz}, 1 \mathrm{H})$, 1.77 (s, 3H); ${ }^{13} \mathrm{C}$ NMR (126 MHz, $\left.\mathrm{CDCl}_{3}\right) \delta: 166.3,163.89$ (d, $J=250.5 \mathrm{~Hz}), 161.83$ (d, $J=246.2 \mathrm{~Hz}), 143.13$ (d, $J=$ $3.1 \mathrm{~Hz}$ ), 140.1, 137.6, 135.6 (d, $J=3.0 \mathrm{~Hz}), 129.0$ (d, $J=$ $8.6 \mathrm{~Hz}), 128.4,127.34$ (d, $J=8.0 \mathrm{~Hz}), 126.4,122.0,121.5$, $115.0(\mathrm{~d}, J=21.6 \mathrm{~Hz}), 114.9$ (d, $J=21.1 \mathrm{~Hz}), 73.6,43.1$, 29.8 .

2,4-双 (4-氯苯基)-2,3-二氢-2-甲基-1H-1,5-苯并二氮 草(3ac): 产率 94\%. 黄色固体, m.p. $143 \sim 147{ }^{\circ} \mathrm{C}$ (lit. ${ }^{[9]}$ m.p. $\left.144 \sim 146{ }^{\circ} \mathrm{C}\right) ;{ }^{1} \mathrm{H}$ NMR $\left(500 \mathrm{MHz}, \mathrm{CDCl}_{3}\right) \delta$ : $7.72 \sim 7.67(\mathrm{~m}, 2 \mathrm{H}), 7.63 \sim 7.59(\mathrm{~m}, 2 \mathrm{H}), 7.55 \sim 7.49(\mathrm{~m}$, $4 \mathrm{H}), 7.33(\mathrm{dd}, J=7.8,1.5 \mathrm{~Hz}, 1 \mathrm{H}), 7.17 \sim 7.14(\mathrm{~m}, 1 \mathrm{H})$, $7.10 \sim 7.07(\mathrm{~m}, 1 \mathrm{H}), 6.89(\mathrm{dd}, J=7.8,1.3 \mathrm{~Hz}, 1 \mathrm{H}), 3.61(\mathrm{~s}$, $1 \mathrm{H}), 3.22(\mathrm{~d}, J=13.5 \mathrm{~Hz}, 1 \mathrm{H}), 2.96(\mathrm{~d}, J=13.5 \mathrm{~Hz}, 1 \mathrm{H})$, 
$1.81(\mathrm{~s}, 3 \mathrm{H}) ;{ }^{13} \mathrm{C}$ NMR (126 MHz, $\left.\mathrm{CDCl}_{3}\right) \delta: 164.3,152.2$, $143.0,138.9,137.3,132.2,131.9,129.4,127.5,127.2$, 126.6, 122.1, 121.3, 118.4, 113.3, 111.2, 73.4, 42.8, 30.0.

2,4-双(4-溴苯基)-2,3-二氢-2-甲基- $1 H$-1,5-苯并二氮 草(3ad): 产率 96\%. 棕色固体, m.p. 144 147 ${ }^{\circ} \mathrm{C}$ (lit. ${ }^{[9]}$ m.p. $\left.148 \sim 150{ }^{\circ} \mathrm{C}\right) ;{ }^{1} \mathrm{H}$ NMR $\left(500 \mathrm{MHz}, \mathrm{CDCl}_{3}\right) \delta$ : $7.51 \sim 7.36(\mathrm{~m}, 8 \mathrm{H}), 7.31(\mathrm{dd}, J=7.6,1.7 \mathrm{~Hz}, 1 \mathrm{H}), 7.13 \sim$ $7.06(\mathrm{~m}, 2 \mathrm{H}), 6.85(\mathrm{dd}, J=7.5,1.5 \mathrm{~Hz}, 1 \mathrm{H}), 3.09$ (d, $J=$ $13.3 \mathrm{~Hz}, 1 \mathrm{H}), 2.91(\mathrm{~d}, J=13.3 \mathrm{~Hz}, 1 \mathrm{H}), 1.75(\mathrm{~s}, 3 \mathrm{H}) ;{ }^{13} \mathrm{C}$ NMR $\left(126 \mathrm{MHz}, \mathrm{CDCl}_{3}\right) \delta: 166.1,146.4,139.9,138.3$, $137.6,131.4,131.3,128.7,128.6,127.4,126.7,124.6$, $122.0,121.5,121.2,73.5,42.9,29.8$.

2,4-双(4-氰基苯基)-2,3-二氢-2-甲基- $1 H$-1,5-苯并二 氮草(3ae): 产率 56\%. 黄色油状物; ${ }^{1} \mathrm{H}$ NMR (500 MHz, $\left.\mathrm{CDCl}_{3}\right) \delta: 7.72 \sim 7.67(\mathrm{~m}, 2 \mathrm{H}), 7.63 \sim 7.59(\mathrm{~m}, 2 \mathrm{H})$, $7.55 \sim 7.49(\mathrm{~m}, 4 \mathrm{H}), 7.33(\mathrm{dd}, J=7.8,1.5 \mathrm{~Hz}, 1 \mathrm{H}), 7.16 \sim$ $7.14(\mathrm{~m}, 1 \mathrm{H}), 7.09 \sim 7.07(\mathrm{~m}, 1 \mathrm{H}), 6.89(\mathrm{dd}, J=7.8,1.3$ $\mathrm{Hz}, 1 \mathrm{H}), 3.61$ (s, 1H), 3.22 (d, $J=13.5 \mathrm{~Hz}, 1 \mathrm{H}), 2.96$ (d, $J$ $=13.5 \mathrm{~Hz}, 1 \mathrm{H}), 1.81(\mathrm{~s}, 3 \mathrm{H}) ;{ }^{13} \mathrm{C} \mathrm{NMR}\left(126 \mathrm{MHz}, \mathrm{CDCl}_{3}\right)$ $\delta: 164.3,152.2,143.0,138.9,137.3,132.2,131.9,129.4$, $127.5,127.2,126.6,122.1,121.3,118.4,113.3,111.2$, 73.4, 42.8, 30.0; HRMS (ESI) calcd for $\mathrm{C}_{24} \mathrm{H}_{19} \mathrm{~N}_{4}[\mathrm{M}+\mathrm{H}]$ 363.1604, found 363.1601 .

2,4-双(4-硝基苯基)-2,3-二氢-2-甲基- $1 H$-1,5-苯并二 氮草 $(3 \mathbf{a f})^{[16]}$ : 产率 $45 \%$. 橙色油状物; ${ }^{1} \mathrm{H}$ NMR (500 $\left.\mathrm{MHz}, \mathrm{CDCl}_{3}\right) \delta: 8.07(\mathrm{~d}, J=8.7 \mathrm{~Hz}, 4 \mathrm{H}), 7.79 \sim 7.67(\mathrm{~m}$, $4 \mathrm{H}), 7.36(\mathrm{dd}, J=7.8,1.5 \mathrm{~Hz}, 1 \mathrm{H}), 7.20 \sim 7.16(\mathrm{~m}, 1 \mathrm{H})$, $7.12 \sim 7.09(\mathrm{~m}, 1 \mathrm{H}), 6.91(\mathrm{dd}, J=7.8,1.2 \mathrm{~Hz}, 1 \mathrm{H}), 3.68(\mathrm{~s}$, $1 \mathrm{H}), 3.32$ (d, $J=13.6 \mathrm{~Hz}, 1 \mathrm{H}), 3.02$ (d, $J=13.6 \mathrm{~Hz}, 1 \mathrm{H})$, $1.85(\mathrm{~s}, 3 \mathrm{H}) ;{ }^{13} \mathrm{C} \mathrm{NMR}\left(126 \mathrm{MHz}, \mathrm{CDCl}_{3}\right) \delta: 163.8,154.0$, $148.4,147.0,144.7,138.8,137.2,129.6,127.8,127.6$, $126.9,123.6,123.4,122.2,121.3,73.3,42.9,30.2$.

2,4-双(4-甲氧羰基苯基)-2,3-二氢-2-甲基- $1 H$ - 1,5 -苯 并二氮草(3ag): 产率 82\%. 橙色油状物; ${ }^{1} \mathrm{H}$ NMR (500 $\left.\mathrm{MHz}, \mathrm{CDCl}_{3}\right) \delta: 7.92 \sim 7.83(\mathrm{~m}, 4 \mathrm{H}), 7.66(\mathrm{~d}, J=8.6 \mathrm{~Hz}$, $2 \mathrm{H}), 7.59(\mathrm{~d}, J=8.6 \mathrm{~Hz}, 2 \mathrm{H}), 7.35 \sim 7.33(\mathrm{~m}, 1 \mathrm{H}), 7.13 \sim$ $7.05(\mathrm{~m}, 2 \mathrm{H}), 6.87(\mathrm{dd}, J=7.7,1.4 \mathrm{~Hz}, 1 \mathrm{H}), 3.89$ (t, $J=6.2$ Hz, 6H), 3.60 (s, 1H), 3.22 (d, J=13.4 Hz, 1H), 2.96 (d, $J=13.4 \mathrm{~Hz}, 1 \mathrm{H}), 1.78(\mathrm{~s}, 3 \mathrm{H}) ;{ }^{13} \mathrm{C} \mathrm{NMR}(126 \mathrm{MHz}$, $\left.\mathrm{CDCl}_{3}\right) \delta: 166.7,166.7,166.0,152.2,143.2,139.6,137.6$, $130.9,129.6,129.3,129.0,128.9,126.9,126.8,125.7$, 121.9, 121.4, 73.8, 52.1, 52.0, 42.9, 30.0; HRMS (ESI) calcd for $\mathrm{C}_{26} \mathrm{H}_{25} \mathrm{~N}_{2} \mathrm{O}_{4}[\mathrm{M}+\mathrm{H}]^{+}$429.1809, found 429.1804 .

2,4-双(4-甲氧基苯基)-2,3-二氢-2-甲基- $1 H$-1,5-苯并 二氮草(3ah): 产率 $80 \%$. 黄色固体, m.p. 115 $118{ }^{\circ} \mathrm{C}$ (lit. ${ }^{[9]}$ m.p. $\left.118 \sim 120{ }^{\circ} \mathrm{C}\right) ;{ }^{1} \mathrm{H}$ NMR (500 MHz, $\left.\mathrm{CDCl}_{3}\right) \delta$ : $7.66 \sim 7.60(\mathrm{~m}, 2 \mathrm{H}), 7.59 \sim 7.52(\mathrm{~m}, 2 \mathrm{H}), 7.36 \sim 7.30(\mathrm{~m}$, $1 \mathrm{H}), 7.10 \sim 7.03(\mathrm{~m}, 2 \mathrm{H}), 6.87 \sim 6.76(\mathrm{~m}, 5 \mathrm{H}), 3.80(\mathrm{~d}, J=$ $19.5 \mathrm{~Hz}, 6 \mathrm{H}), 3.45$ (s, 1H), 3.07 (d, $J=13.2 \mathrm{~Hz}, 1 \mathrm{H}), 2.93$ $(\mathrm{d}, J=13.2 \mathrm{~Hz}, 1 \mathrm{H}), 1.75(\mathrm{~s}, 3 \mathrm{H}) ;{ }^{13} \mathrm{C} \mathrm{NMR}(126 \mathrm{MHz}$, $\mathrm{CDCl}_{3}$ ) $\delta: 167.1,161.0,158.5,140.7,140.1,138.0,132.3$, $128.8,128.2,126.6,125.9,121.8,121.5,113.5,113.4$, $73.3,55.3,55.3,42.8,29.7$.

2,4-双(4-甲基苯基)-2,3-二氢-2-甲基- $1 H$-1,5-苯并二 氮草(3ai): 产率 90\%. 黄色固体, m.p. 98 99 ${ }^{\circ} \mathrm{C}$ (lit. ${ }^{[9]}$ m.p. 99 $\left.100{ }^{\circ} \mathrm{C}\right) ;{ }^{1} \mathrm{H}$ NMR $\left(500 \mathrm{MHz}, \mathrm{CDCl}_{3}\right) \delta: 7.58(\mathrm{~d}$, $J=8.2 \mathrm{~Hz}, 2 \mathrm{H}), 7.50(\mathrm{~d}, J=8.2 \mathrm{~Hz}, 2 \mathrm{H}), 7.36 \sim 7.31(\mathrm{~m}$, $1 \mathrm{H}), 7.14 \sim 7.02(\mathrm{~m}, 6 \mathrm{H}), 6.86 \sim 6.81(\mathrm{~m}, 1 \mathrm{H}), 3.53(\mathrm{~s}$, $1 \mathrm{H}), 3.09$ (d, $J=13.2 \mathrm{~Hz}, 1 \mathrm{H}), 2.99$ (d, $J=13.2 \mathrm{~Hz}, 1 \mathrm{H})$, $2.34(\mathrm{~d}, J=17.9 \mathrm{~Hz}, 6 \mathrm{H}), 1.75(\mathrm{~s}, 3 \mathrm{H}) ;{ }^{13} \mathrm{C}$ NMR $(126$ $\left.\mathrm{MHz}, \mathrm{CDCl}_{3}\right) \delta: 167.6,145.1,140.4,140.0,138.3,137.0$, $136.7,129.0,128.8,128.5,127.2,126.1,125.2,121.6$, 121.4, 73.3, 42.9, 29.9, 21.3, 20.9.

2,4-双([1,1'-联苯]-4-基)-2,3-二氢-2-甲基- $1 H$-1,5-苯 并二氮草(3aj): 产率 84\%. 橙色油状物; ${ }^{1} \mathrm{H}$ NMR (500 $\left.\mathrm{MHz}, \mathrm{CDCl}_{3}\right) \delta: 7.71(\mathrm{~d}, J=8.2 \mathrm{~Hz}, 4 \mathrm{H}), 7.59 \sim 7.31(\mathrm{~m}$, $16 \mathrm{H}), 7.15 \sim 7.03(\mathrm{~m}, 2 \mathrm{H}), 6.90(\mathrm{dd}, J=7.5,1.6 \mathrm{~Hz}, 1 \mathrm{H})$, $3.60(\mathrm{~s}, 1 \mathrm{H}), 3.22(\mathrm{~d}, J=13.2 \mathrm{~Hz}, 1 \mathrm{H}), 3.06(\mathrm{~d}, J=13.2$ $\mathrm{Hz}, 1 \mathrm{H}), 1.85(\mathrm{~s}, 3 \mathrm{H}) ;{ }^{13} \mathrm{C} \mathrm{NMR}\left(126 \mathrm{MHz}, \mathrm{CDCl}_{3}\right) \delta$ : $167.3,146.7,142.4,140.7,140.4,140.2,140.1,138.5$, $138.1,128.8,128.8,128.7,127.6,127.6,127.3,127.1$, 127.1, 127.1, 126.7, 126.4, 126.0, 121.8, 121.5, 73.6, 43.1, 29.9; HRMS (ESI) calcd for $\mathrm{C}_{34} \mathrm{H}_{29} \mathrm{~N}_{2}[\mathrm{M}+\mathrm{H}]{ }^{+}$ 465.2325, found 465.2329.

2,4-双(4-苯甲酰基苯基)-2,3-二氢-2-甲基- $1 H$-1,5-苯 并二氮草(3ak): 产率 $86 \%$. 橙色油状物; ${ }^{1} \mathrm{H}$ NMR (500 $\left.\mathrm{MHz}, \mathrm{CDCl}_{3}\right) \delta: 7.75 \sim 7.62(\mathrm{~m}, 12 \mathrm{H}), 7.61 \sim 7.53(\mathrm{~m}$, 2H), 7.45 (dd, $J=15.1,7.4 \mathrm{~Hz}, 4 \mathrm{H}), 7.37$ (dd, $J=7.7,1.5$ $\mathrm{Hz}, 1 \mathrm{H}), 7.16 \sim 7.07(\mathrm{~m}, 2 \mathrm{H}), 6.92(\mathrm{dd}, J=7.7,1.2 \mathrm{~Hz}$, 1H), 3.65 (s, 1H), 3.32 (d, $J=13.3 \mathrm{~Hz}, 1 \mathrm{H}), 3.04$ (d, $J=$ $13.3 \mathrm{~Hz}, 1 \mathrm{H}), 1.86(\mathrm{~s}, 3 \mathrm{H}) ;{ }^{13} \mathrm{C}$ NMR $\left(126 \mathrm{MHz}, \mathrm{CDCl}_{3}\right) \delta$ : $196.3,196.1,166.1,151.6,142.9,139.4,138.2,137.7$, $137.5,137.4,136.4,132.6,132.5,130.1,130.0,129.9$, $129.8,129.1,128.3,128.3,127.1,126.8,125.7,121.8$, 121.3, 73.9, 43.1, 30.0; HRMS (ESI) calcd for $\mathrm{C}_{36} \mathrm{H}_{29} \mathrm{~N}_{2} \mathrm{O}_{2}$ $[\mathrm{M}+\mathrm{H}]^{+}$521.2224, found 521.2218.

2,4-双(3-溴苯基)-2,3-二氢-2-甲基- $1 H$-1,5-苯并二氮 草 $(3 \mathrm{al}){ }^{[17]}$ : 产率 $74 \%$. 黄色固体, m.p. $148 \sim 150{ }^{\circ} \mathrm{C}$ (lit. ${ }^{[16]}$ m.p. $\left.150 \sim 153{ }^{\circ} \mathrm{C}\right) ;{ }^{1} \mathrm{H} \mathrm{NMR}\left(500 \mathrm{MHz}, \mathrm{CDCl}_{3}\right) \delta$ : 
$7.77(\mathrm{t}, J=1.8 \mathrm{~Hz}, 1 \mathrm{H}), 7.66(\mathrm{t}, J=1.8 \mathrm{~Hz}, 1 \mathrm{H}), 7.55 \sim$ $7.41(\mathrm{~m}, 3 \mathrm{H}), 7.37 \sim 7.30(\mathrm{~m}, 2 \mathrm{H}), 7.16 \sim 7.06(\mathrm{~m}, 4 \mathrm{H})$, $6.86(\mathrm{dd}, J=7.5,1.6 \mathrm{~Hz}, 1 \mathrm{H}), 3.45(\mathrm{~s}, 1 \mathrm{H}), 3.07$ (d, $J=$ $13.3 \mathrm{~Hz}, 1 \mathrm{H}), 2.89$ (d, $J=13.3 \mathrm{~Hz}, 1 \mathrm{H}), 1.75$ (s, 3H); ${ }^{13} \mathrm{C}$ NMR $\left(126 \mathrm{MHz}, \mathrm{CDCl}_{3}\right) \delta: 165.8,149.4,141.2,139.7$, $137.5,132.6,130.2,130.0,129.9,129.5,129.0,128.6$, $126.7,125.4,124.3,122.7,122.4,122.0,121.5,73.7,43.0$, 29.6 .

2,4-二(2-噻吩基)-2,3-二氢-2-甲基-1H-1,5-苯并二氮 草(3am): 产率 56\%. 棕色固体, m.p. 92 94 ${ }^{\circ} \mathrm{C}$ (lit. ${ }^{[9]}$ m.p. $\left.90 \sim 91{ }^{\circ} \mathrm{C}\right) ;{ }^{1} \mathrm{H}$ NMR $\left(500 \mathrm{MHz}, \mathrm{CDCl}_{3}\right) \delta$ : $7.61(\mathrm{dd}$, $J=5.0,1.3 \mathrm{~Hz}, 1 \mathrm{H}), 7.34 \sim 7.23(\mathrm{~m}, 5 \mathrm{H}), 7.19(\mathrm{dd}, J=5.0$, $1.4 \mathrm{~Hz}, 1 \mathrm{H}), 7.12 \sim 7.05(\mathrm{~m}, 2 \mathrm{H}), 6.84 \sim 6.79(\mathrm{~m}, 1 \mathrm{H}), 3.48$ (s, 1H), 2.99 (d, J=13.2 Hz, 1H), 2.89 (d, $J=13.2 \mathrm{~Hz}$, $1 \mathrm{H}), 1.76(\mathrm{~s}, 4 \mathrm{H}) ;{ }^{13} \mathrm{C}$ NMR $\left(126 \mathrm{MHz}, \mathrm{CDCl}_{3}\right) \delta: 163.6$, $149.8,143.7,141.0,137.6,127.8,126.7,126.4,126.3$, 126.0, 125.6, 125.6, 122.4, 121.8, 120.6, 72.4, 43.8, 29.8 .

2,4-二正己基-2,3-二氢-2-甲基- $1 H$-1,5-苯并二氮草 (3an) ${ }^{[9]}$ : 产率 $47 \%$. 黄色油状物; ${ }^{1} \mathrm{H}$ NMR (500 MHz, $\left.\mathrm{CDCl}_{3}\right) \delta: 7.18 \sim 7.12(\mathrm{~m}, 1 \mathrm{H}), 7.01 \sim 6.94(\mathrm{~m}, 2 \mathrm{H})$, $6.76 \sim 6.67(\mathrm{~m}, 1 \mathrm{H}), 2.62 \sim 2.53(\mathrm{~m}, 2 \mathrm{H}), 2.24(\mathrm{~d}, J=12.9$ $\mathrm{Hz}, 1 \mathrm{H}), 2.16(\mathrm{~d}, J=12.9 \mathrm{~Hz}, 1 \mathrm{H}), 1.71$ (dt, $J=15.5,7.5$ $\mathrm{Hz}, 2 \mathrm{H}), 1.66 \sim 1.52(\mathrm{~m}, 2 \mathrm{H}), 1.46 \sim 1.25(\mathrm{~m}, 17 \mathrm{H}), 0.97 \sim$ $0.84(\mathrm{~m}, 6 \mathrm{H}) ;{ }^{13} \mathrm{C} \mathrm{NMR}\left(126 \mathrm{MHz}, \mathrm{CDCl}_{3}\right) \delta: 175.3$, $140.8,137.9,127.0,125.4,121.8,121.7,70.7,43.4,42.9$, $42.5,31.8,31.7,29.8,29.2,27.6,26.5,24.2,22.6,14.1$, 14.0 .

\section{2,4-二环丙基-2,3-二氢-2-甲基- $1 H$-1,5-苯并二氮草} (3ao) $)^{[9]}$ : 产率 $40 \%$. 黄色油状物; ${ }^{1} \mathrm{H}$ NMR (500 MHz, $\left.\mathrm{CDCl}_{3}\right) \delta: 7.10 \sim 7.02(\mathrm{~m}, 1 \mathrm{H}), 6.97 \sim 6.90(\mathrm{~m}, 2 \mathrm{H})$, $6.72 \sim 6.68(\mathrm{~m}, 1 \mathrm{H}), 2.85(\mathrm{~s}, 1 \mathrm{H}), 2.37(\mathrm{~d}, J=12.8 \mathrm{~Hz}$, $1 \mathrm{H}), 2.29(\mathrm{~d}, J=12.8 \mathrm{~Hz}, 1 \mathrm{H}), 1.86(\mathrm{tt}, J=8.1,4.8 \mathrm{~Hz}$, $1 \mathrm{H}), 1.23 \sim 1.08(\mathrm{~m}, 6 \mathrm{H}), 1.00 \sim 0.88(\mathrm{~m}, 2 \mathrm{H}), 0.63(\mathrm{td}$, $J=9.8,5.5 \mathrm{~Hz}, 1 \mathrm{H}), 0.53 \sim 0.39(\mathrm{~m}, 2 \mathrm{H}), 0.28(\mathrm{td}, J=9.9$, $5.3 \mathrm{~Hz}, 1 \mathrm{H}) ;{ }^{13} \mathrm{C} \mathrm{NMR}\left(126 \mathrm{MHz}, \mathrm{CDCl}_{3}\right) \delta: 175.8,140.2$, $137.84,127.0,125.0,121.5,121.4,69.9,43.7,26.6,22.5$, $20.6,9.9,9.5,1.3,0.5$.

辅助材料(Supporting Information) 产物 3aa $\sim 3 a 0$ 的 核磁共振表征图谱. 这些材料可以免费从本刊网站 (http://sioc-journal.cn/)上下载.

\section{References}

[1] (a) Randall, L. O.; Kappel, B. In Benzodiazepines, Eds.: Garattini, S.; Mussini, E.; Randall, L. O., Raven Press, New York, 1973, p. 27. (b) Smalley, R. K. In Comprehensive Organic Chemistry, Eds.: Barton, D.; Ollis, W. D., Pergamon, Oxford, 1979, Vol. 4, p. 600. (c) Schutz, H. Benzodiazepines, Springer, Heidelberg, 1982. (d) Landquist, J. K. In Comprehensive Heterocyclic Chemistry, Eds.: Katritzky, A. R.; Rees, C. W., Pergamon, Oxford, 1984, Vol. 1 , pp. $166 \sim 170$

[2] Leyva-Perez, A.; Cabrero-Antonino, J. R.; Corma, A. Tetrahedron 2010, 66, 8203 and references cited therein.

[3] Fad, L. D.; Bethell, R.; Bonneau, P.; Bös, M.; Bousquet, Y.; Coordingley, M. G.; Coulombe, R.; Deroy, P.; Faucher, A.-M.; Gagnon, A.; Goudreau, N.; Grand-Maître, C.; Guse, I.; Hucke, O.; Kawai, S. H.; Lacoste, J.-E.; Landry, S.; Lemke, C. T.; Malenfant, E.; Mason, S.; Morin, S.; O’Meara, J.; Simoneau, B.; Titolo, S.; Yoakim, C. Bioorg. Med. Chem. Lett. 2011, 21, 398.

[4] (a) El-snyed, A. M.; Abdel-ghany, H.; El-snghier, A. M. M. Synth. Commun. 1999, 29, 3561.

(b) Essaber, M.; Baouid, A.; Hasnaoui, A.; Benharref, A.; Lavergne, J. P. Synth. Commun. 1998, 28, 4097.

(c) Reddy, K. V. V; Rao, P. S.; Ashok, D. Synth. Commun. 2000, 30, 1825 .

[5] (a) Pan, X.Q.; Zou, J. P.; Huang, Z. H.; Zhang, W. Tetrahedron Lett. 2008, 49, 5302.

(b) Nardi, M.; Cozza, A.; Maiuolo, L.; Oliverio, M.; Procopio, A Tetrahedron Lett. 2011, 52, 4827 and references cited therein. (c) Feng, S.-E.; Xu, F.; Shen, Q. Chin. J. Chem. 2008, 26, 861

[6] Ried, W.; Torinus, E. Chem. Ber. 1959, 92, 2902.

[7] (a) Neochoritis, C. G.; Tsoleridis, C. A.; Sephanidou-Stephanatou, J.; Kontogiorgis, C. A.; Hadjipavlou-Litina, D. J. J. Med. Chem. 2010, 53, 8409 .

(b) Ha, S. K.; Shobha, D.; Moon, E.; Chari, M. A.; Mukkanti, K.; Kim, S. H.; Ahn, K. H.; Kim, S. Y. Bioorg. Med. Chem. Lett. 2010, 20, 3969.

(c) Climent, M. J.; Corma, A.; Iborra, S.; Santos, L. L. Chem.-Eur. J. 2009, 15, 8834 .

[8] Maiti, G.; Kayal, U.; Karmakar, R.; Bhattacharya, R. N. Tetrahedron Lett. 2012, 53, 1460.

[9] Qian, J.; Liu, Y.; Cui, J.; Xu, Z. J. Org. Chem. 2012, 77, 4484

[10] Guo, P.; Zeng, X.; Chen, S.; Luo, M. J. Organomet. Chem. 2014, $751,438$.

[11] (a) Cai, R.; Ye, X.; Sun, Q.; He, Q.; He, Y.; Ma, S.; Shi, X. ACS Catal. 2017, 7, 1087.

(b) Young, P. C.; Green, S. L. J.; Rosair, G. M.; Lee, A.-L. Dalton Trans. 2013, 42, 9645.

[12] Chen, L.; Chen, K.; Zhu, S. Chem 2018, 4, 1208.

[13] (a) McCahill, J. S. J.; Welch, G. C.; Stephan, D. W. Angew. Chem., Int. Ed. 2007, 46, 4968.

(b) Stephan, D. W. Acc. Chem. Res. 2015, 48, 306.

[14] (a) Wang, Y.; Wang, Z.; Li, Y.; Wu, G.; Cao, Z.; Zhang, L. Nat. Commun. 2014, 5, 3687.

(b) Li, X.; Liao, S.; Wang, Z.; Zhang, L. Org. Lett. 2017, 19, 3687.

(c) Wang, Z.; Hervieu, C.; Wong, Y.-F.; Zanoni, G.; Zhang, L. J. Am. Chem. Soc. 2017, 139, 16064.

[15] (a) Liu, Y.; Qian, J.; Lou, S.; Xu, Z. J. Org. Chem. 2010, 75, 6300 .

(b) Liu, Y.; Qian, J.; Lou, S.; Zhu, J.; Xu, Z. J. Org. Chem. 2010, $75,1309$.

(c) Liu, Y.; Zhu, J.; Qian, J.; Jiang, B.; Xu, Z. J. Org. Chem. 2011, 76, 9096.

(d) Qian, J.; Liu, Y.; Zhu, J.; Jiang, B.; Xu, Z. Org. Lett. 2011, 13, 4220 .

(e) Liu, Y.; Zhu, J.; Qian, J.; Xu, Z. J. Org. Chem. 2012, 77, 5411.

[16] Patil, V. D.; Patil, K. P.; Sutar, N. R.; Gidh, P. V. Heterocycl. Lett. 2016, 6,61 .

[17] Jeganathan, M.; Pitchumani, K. ACS Sustainable Chem. Eng. 2014, 2,1169 